\title{
Nonlocal Physics in the Wave Function Terminology
}

\author{
Boris V. Alexeev \\ MIREA-Russian State Technological University, Moscow, Russia \\ Email: Boris.Vlad.Alexeev@gmail.com
}

How to cite this paper: Alexeev, B.V. (2021) Nonlocal Physics in the Wave Function Terminology. Journal of Applied Mathematics and Physics, 9, 2889-2908. https://doi.org/10.4236/jamp.2021.911183

Received: October 9, 2021

Accepted: November 16, 2021

Published: November 19, 2021

Copyright $\odot 2021$ by author(s) and Scientific Research Publishing Inc. This work is licensed under the Creative Commons Attribution International License (CC BY 4.0).

http://creativecommons.org/licenses/by/4.0/

\begin{abstract}
Shortcomings of the Boltzmann physical kinetics and the Schrödinger wave mechanics are considered. From the position of nonlocal physics, the Schrödinger equation is a local equation; this fact leads to the great shortcomings of the linear Schrödinger wave mechanics. Nonlocal nonlinear quantum mechanics is considered using the wave function terminology.
\end{abstract}

\section{Keywords}

Nonlocal Physics, Transport Processes in Quantum Mechanics, Shortcomings of the Schrödinger Equation, Wave Function Terminology

\section{Introduction. Shortcomings of the Schrödinger and Madelung Quantum Mechanics}

Shortcomings of the Boltzmann physical kinetics consist in the local description of the transport processes on the level of infinitely small physical volumes (PhSV) as elements of diagnostics. In other words, in Boltzmann's theory [1] PhSV is a closed thermodynamic system. All details of the proposed nonlocal theory can be found in [2]-[10]. But here we formulate the remarks of the principal significance:

1) Kinetic theory and hydrodynamic theory should be non-local.

2) The nonlocal effects are due to the reduced description and are not related to the specific division of the physical system by the PhSV grid.

3) Accurate derivation of the kinetic equation with respect to the one-particle distribution function (DF) should lead to corrections of the order of the Knudsen number before uncoupling the Bogolyubov chain [2]-[10].

4) This means that in the Boltzmann equation the terms of the order of the Knudsen number are lost, significant for both large and small Knudsen num- 
bers.

5) The Boltzmann equation does not even belong to the class of minimal models, being only a "plausible" equation.

6) Boltzmann equation in this sense-the wrong equation.

7) Obviously the mentioned non-local effects can be discussed from viewpoint of breaking of the Bell's inequalities because in the non-local theory the measurement (realized in $\mathrm{PhSV}_{1}$ ) has an influence on the measurement realized in the adjoining space-time point in $\mathrm{PhSV}_{2}$ and verse versa.

8) Madelung's quantum hydrodynamics is equivalent to the Schrödinger Equation (SE) and furnishes the description of the quantum particle evolution in the form of Euler equation and continuity equation. Madelung quantum hydrodynamics does not lead to the energy equation in principal, and SE leads to the soliton destruction in the Schrödinger-Madelung wave mechanics.

9) Nonlocal physical kinetics brings the strict approximation of non-local effects in space and time and after transfer to the local approximation leads to parameter $\tau$, which on the quantum level corresponds to the uncertainty principle "time-energy". Methods of the $\tau$ definition in [2]-[10] are considered.

It is established that the theory of transport processes (including quantum mechanics) can be presented within the framework of the universal theory (unified theory of dissipative systems) based on the nonlocal physical description [2]-[10]. It is shown, in particular, that the equations of nonlocal physics lead to the appearance of solitons, which supports the Schrödinger opinion, who interpreted quantum mechanics from the point of view of the existence of waves of matter.

Let us turn to the logic of the development of the non-local theory of transport processes:

1) In 1926 Madelung published a brilliant article [11] in which he transformed the quantum postulate (Schrödinger equation containing the $\psi$ wave function) in hydrodynamics. In other words, the evolution of a single bound electron was possible to interpret as some effective flow.

2) In 1964 John Stewart Bell [12] found that local statistical theory of dissipative processes is incorrect in principle.

3) In 2007 I found that the Schrödinger equation and hydrodynamic Madelung's form are a deep particular case of nonlocal kinetic equations (see for example [9] [10]) as a result of the transition to the local limit of non-local equations. In other words, generalized hydrodynamic equations (GHE) should contain Schrödinger Equation (SE) as a deep special case. This affirmation was proved in articles [9] [10]; we formulated in explicit form all assumptions (all steps) that should be implemented to obtain SE from GHE.

This means that generalized physical kinetics (as created earlier by me, see for example [2]-[10]) has been extended to quantum physics in the form of non-local quantum hydrodynamics (NLQH). Then a new quantum mechanics of dissipative processes has been created. 
The Schrödinger equation is not dissipative. Therefore, generalized quantum hydrodynamics is a tool for solving problems in the theory of dissipative nano-systems.

Here just note that:

1) $S E$ is not able to give a self-consistent description of the nucleus-electron shell.

2) SE does not lead to an independent analogue of the hydrodynamic energy equation.

3) SE (and its equivalent hydrodynamic Madelung form) is not a dissipative equation and therefore cannot be applied to the description of dissipative processes in nanotechnology.

4) The Schrödinger equation cannot explain spontaneous emission, since the wave function of the excited state is an exact solution of the time-dependent SE equation [13].

5) The linear Schrödinger equation cannot describe the measurement process in quantum mechanics, since the measurement process is nonlinear, stochastic and irreversible in time.

6) The Schrödinger equation cannot describe the processes of mutual transformations of elementary particles.

7) The Schrödinger equation is not able to describe the "nucleus-electron shell" complex as a whole.

8) The Schrödinger equation is not able to describe the spatial electron shell without using additional assumptions, such as the Pauli principle.

9) To a large extent, the result of quantization is the result of cutting off infinite series and turning them into polynomials. This process resembles the transformation of a traveling wave into a system of standing waves if a reflection from an obstacle is introduced.

As you know, the basic equation of quantum mechanics-the Schrödinger equation is written in terms of the wave function and is, in fact, a postulate. The Schrödinger equation is "guessed" based on reasonable physical considerations. The main stages of similar "derivation" can be characterized as follows:

1) The desired equation should reflect the wave properties of particles, including one-dimensional harmonic oscillations

$$
\xi=A \cos (\omega t-k x)
$$

Relation (1.1) includes the circular frequency $\omega$ and wave number $k=2 \pi / \lambda$, where $\lambda$ is the wavelength. In accordance with the de Broglie principle, we compare this oscillation to a certain corpuscular object and introduce the connection of its kinetic energy $E_{\kappa}$ and momentum $p$ with the frequency attributed to it:

$$
\begin{gathered}
\omega=2 \pi v, \\
p=\frac{h v}{c}=\frac{h}{\lambda} .
\end{gathered}
$$

Formulas (1.2), (1.3) are known from the theory of radiation characteristics of 
photons, distributed in all material particles.

2) Now we introduce a complex function $\psi(x, t)$ as a characteristic of the field associated with the existence of this object:

$$
\psi=\mathrm{e}^{-i(\omega t-k x)} .
$$

Let us note, that

$$
\omega=E_{\kappa} / \hbar, \quad k=2 \pi / \lambda=p / \hbar .
$$

3) After substitution (1.5) in (1.4) one obtains:

$$
\psi=\exp \left[-\frac{i}{\hbar}\left(E_{\kappa} t-p x\right)\right] .
$$

Let us differentiate the wave function (1.6) by time

$$
\frac{\partial \psi}{\partial t}=\psi\left(-i \frac{E_{\kappa}}{\hbar}\right)
$$

and multiply both parts of (1.7) by i

$$
i \hbar \frac{\partial \psi}{\partial t}=E_{\kappa} \psi \text {. }
$$

Now let's differentiate (1.6) twice by $x$

$$
\frac{\partial^{2} \psi}{\partial x^{2}}=\psi\left(i \frac{p}{\hbar}\right)^{2}=-\frac{p^{2}}{\hbar^{2}} \psi .
$$

when differentiating, it is assumed that the kinetic energy and momentum of the particle do not depend on the coordinates and time, and, therefore, we are talking about a free particle. Because

$$
E_{\kappa}=\frac{p^{2}}{2 m},
$$

it is possible to record (1.9) in the form of

$$
-\frac{\hbar^{2}}{2 m} \frac{\partial^{2} \psi}{\partial x^{2}}=E_{\kappa} \psi .
$$

Compare now (1.8) and (1.11), the right parts of these relations are the same. Therefore, recording is possible

$$
i \hbar \frac{\partial \psi}{\partial t}=-\frac{\hbar^{2}}{2 m} \frac{\partial^{2} \psi}{\partial x^{2}} .
$$

Equation (1.12) is declared to be the sought equation describing the state of the considered corpuscle.

4) The following reasonable generalization:

a) if we consider $3 \mathrm{D}$ case:

$$
i \hbar \frac{\partial \psi}{\partial t}=-\frac{\hbar^{2}}{2 m}\left(\frac{\partial^{2} \psi}{\partial x^{2}}+\frac{\partial^{2} \psi}{\partial y^{2}}+\frac{\partial^{2} \psi}{\partial z^{2}}\right) .
$$

b) if we consider now the particle movement in a potential field $U(x, y, z, t)$; this potential energy should be added on the right-hand side of (1.13): 


$$
i \hbar \frac{\partial \psi}{\partial t}=-\frac{\hbar^{2}}{2 m}\left(\frac{\partial^{2} \psi}{\partial x^{2}}+\frac{\partial^{2} \psi}{\partial y^{2}}+\frac{\partial^{2} \psi}{\partial z^{2}}\right)+U \psi
$$

Equation (1.14) was derived in 1926 by Erwin Schrödinger and bears his name. Schrödinger's first message [14] is entitled "Quantization as an eigenvalue problem."

Some comments to this equation:

1) Restriction on the mass of the particle is not entered. However, the particle is considered as a material point. Generalization of the equation to many-particle systems is a separate problem. To solve, for example, the problem of the evolution of a system containing $n$ electrons, it is necessary to consider $\psi$ as a function of $3 n$ independent coordinates and time $t$.

2) The Schrödinger equation belongs to the class of linear equations. This means that in the sum of some solutions $\psi_{1}$ and $\psi_{2}$ of the Schrödinger equation there is also a solution to this equation $\psi=\psi_{1}+\psi_{2}$.

3) The Schrödinger equation belongs to the class of reversible equations of physics; the form of this equation does not change when replacing $t$ with $(-t)$ and simultaneously $\psi$ replacing the wave functions on a complex conjugate wave function $\psi^{*}$.

4) The Schrödinger equation does not satisfy Lorentz transformation and therefore does not describe relativistic phenomena. In particular, the Schrödinger equation does not contain the spin of elementary particles, which is a consequence of the relativistic quantum Dirac equation. No calculations are necessary to ensure that Equation (1.14) is not invariant with respect to the Lorenz's transformation. As it is known, in the four-dimensional Minkowski space relativistic equations contain "equal standing" for independent variables, including time. However, the Schrödinger equation contains the second derivatives of coordinates and only the first derivatives of time.

The disadvantages of the Schrödinger equation:

1) The Schrödinger equation is a postulate. Another differentiation of the function leads to other equations, for example, to equations containing the second derivative of time.

2) The Schrödinger equation does not describe dissipative processes.

3) The Schrödinger equation is not able to describe the whole complex "nucleus-electron shell".

4) The Schrödinger equation is unable to describe a spatial electron shell without the use of additional assumptions such as the Pauli principle.

5) To a large extent, the quantization result is the result of cutting infinite series and turning them into polynomials.

The next obvious step was taken by E. Madelung (E. Madelung [11]) in 1926. We are talking about the derivation of a special form of the Schrödinger equation after the wave function representation in the form of

$$
\psi(x, y, z)=\alpha(x, y, z) \mathrm{e}^{i \beta(x, y, z)} .
$$


After using (1.15) and separating the real and imaginary parts of Equation (1.15), Madelung obtained two hydrodynamic equations-the continuity equation

$$
\frac{\partial \rho}{\partial t}+\frac{\partial}{\partial \mathbf{r}} \cdot(\rho \mathbf{v})=0
$$

and the equation of motion for the Euler potential flow

$$
\frac{\partial \mathbf{v}}{\partial t}+\left(\mathbf{v} \cdot \frac{\partial}{\partial \mathbf{r}}\right) \mathbf{v}=-\frac{1}{m} \frac{\partial}{\partial \mathbf{r}} U^{*}
$$

where the effective potential has the form

$$
U^{\bullet}=U+U_{q u}-\frac{\hbar^{2}}{2 m \sqrt{\rho}} \Delta \sqrt{\rho}=U-\frac{\hbar^{2}}{4 m \sqrt{\rho}}\left[\Delta \rho-\frac{1}{2 \rho}\left(\frac{\partial \rho}{\partial \mathbf{r}}\right)^{2}\right] .
$$

Identifications

$$
\begin{gathered}
\rho=\alpha^{2} \\
\mathbf{v}=\frac{\partial}{\partial \mathbf{r}}\left(\frac{\beta \hbar}{m}\right)
\end{gathered}
$$

introduced in Equations (1.16), (1.17). The existence of a condition (1.20) means that the stream has potential $\frac{\beta \hbar}{m}$.

Schrödinger assumed, that the spatial evolution of a quantum object (such as an electron) can be described as the motion of a wave packet, in other words, a soliton. But Pauli showed that the wave packet, built on Schrödinger, is spreading in space.

Really, the non-stationary 1D Schrödinger Equation (SE) can be writes as

$$
i \hbar \frac{\partial \psi}{\partial t}=-\frac{\hbar^{2}}{2 m} \Delta \psi
$$

where $\psi$ is the wave function. In the ID case we have

$$
i \frac{\partial \psi}{\partial t}=-\frac{\hbar}{2 m} \frac{\partial^{2} \psi}{\partial x^{2}} .
$$

Let us introduce the scales $t_{0}, x_{0}=\sqrt{\frac{\hbar}{m} t_{0}}$. Introducing the dimensionless time $\tilde{t}$ and Cartesian distance $\tilde{x}$, we find for $(1.22)\left(\hbar=1.054572 \times 10^{-27} \mathrm{erg} \cdot \mathrm{s}\right)$

$$
i \frac{\partial \psi}{\partial \tilde{t}}=-\frac{1}{2} \frac{\partial^{2} \psi}{\partial \tilde{x}^{2}} .
$$

The solution (1.23) has the form

$$
\psi(\tilde{x}, \tilde{t})=\frac{\sqrt[4]{\frac{2}{\pi}}}{\sqrt{1+2 i \tilde{t}}} \mathrm{e}^{-\frac{1}{4} \tilde{\tilde{k}}_{0}^{2}} \mathrm{e}^{-\frac{1}{1+2 i \tilde{t}}\left(\tilde{x}-\frac{i \tilde{k}_{0}}{2}\right)^{2}},
$$

if

$$
\psi(\tilde{x}, 0)=\sqrt[4]{\frac{2}{\pi}} \mathrm{e}^{-\frac{1}{4} \tilde{k}_{0}^{2}} \mathrm{e}^{-\left(\tilde{x}-\frac{i \tilde{k}_{0}}{2}\right)^{2}},
$$


where $\tilde{k}_{0}$ is the dimensionless wave number. We notice also that

$$
\psi(\tilde{x}, 0)=\sqrt[4]{\frac{2}{\pi}} \mathrm{e}^{-\frac{1}{4} \tilde{k}_{0}^{2}} \mathrm{e}^{-\left(\tilde{x}^{2}-\tilde{\tilde{x}_{0}} i-\frac{\tilde{k}_{0}^{2}}{4}\right)}
$$

The validity (1.24) can be proved after substitution (1.24) into (1.23). For example

$$
\begin{aligned}
i \frac{\partial \psi}{\partial \tilde{t}}= & \frac{\sqrt[4]{\frac{2}{\pi}}}{(1+2 i \tilde{t})^{3 / 2}} \mathrm{e}^{-\frac{1}{4} \tilde{k}_{0}^{2}} \mathrm{e}^{-\frac{1}{1+2 i \tilde{t}}\left(\tilde{x}-\frac{i \tilde{k}_{0}}{2}\right)^{2}} \\
& -\frac{\sqrt[4]{\frac{2}{\pi}}}{(1+2 i \tilde{t})^{1 / 2}} \mathrm{e}^{-\frac{1}{4} \tilde{k}_{0}^{2}} \mathrm{e}^{-\frac{1}{1+2 i \tilde{t}}\left(\tilde{x}-\frac{i \tilde{k}_{0}}{2}\right)^{2}}\left[\left(\tilde{x}-\frac{i \tilde{k}_{0}}{2}\right)^{2} \frac{2}{(1+2 i \tilde{t})^{2}}\right]
\end{aligned}
$$

or

$$
i \frac{\partial \psi}{\partial \tilde{t}}=\frac{\sqrt[4]{\frac{2}{\pi}}}{(1+2 i \tilde{t})^{3 / 2}} \mathrm{e}^{-\frac{1}{4} \tilde{k}_{0}^{2}} \mathrm{e}^{-\frac{1}{1+2 i \tilde{t}}\left(\tilde{x}-\frac{i \tilde{k}_{0}}{2}\right)^{2}}\left[1-\frac{2}{1+2 i \tilde{t}}\left(\tilde{x}-\frac{i \tilde{k}_{0}}{2}\right)^{2}\right]
$$

After calculation we find

$$
\frac{\partial^{2}}{\partial \tilde{x}^{2}} \psi(\tilde{x}, \tilde{t})=-\frac{2 \sqrt[4]{\frac{2}{\pi}}}{(1+2 i \tilde{t})^{\frac{3}{2}}} \mathrm{e}^{-\frac{1}{4} \tilde{k}_{0}^{2}} \mathrm{e}^{-\frac{1}{1+2 i \tilde{t}}\left(\tilde{x}-\frac{\tilde{i} \tilde{k}_{0}}{2}\right)^{2}}\left\{1-\frac{2}{1+2 i \tilde{t}}\left(\tilde{x}-\frac{i \tilde{k}_{0}}{2}\right)^{2}\right\}
$$

and comparing (1.29) and (1.28) we find identity (1.23).

Let us find now $|\psi(\tilde{x}, \tilde{t})|^{2}=\psi \psi^{*}$. With this aim we should obtain the complex conjugate value $\psi^{*}$. We have the transformed wave function $\psi$

$$
\begin{aligned}
& \left.\psi(\tilde{x}, \tilde{t})=\frac{\sqrt[4]{\frac{2}{\pi}}}{\sqrt{1+2 i \tilde{t}}} \mathrm{e}^{-\frac{1}{4} \tilde{k}_{0}^{2}} \mathrm{e}^{-\frac{1}{1+4 \tilde{t}^{2}}\left[\tilde{x}^{2}-\frac{\tilde{k}_{0}^{2}}{4}-2 i \tilde{x} \tilde{k}_{0}-2 i \tilde{t}\left(\tilde{x}^{2}-\frac{\tilde{k}_{0}^{2}}{4}-2 i i \tilde{x}_{0}\right)\right.}\right] \\
& \left.=\frac{\sqrt[4]{\frac{2}{\pi}}}{\sqrt{1+4 \tilde{t}^{2}}} \mathrm{e}^{-\frac{1}{4} \tilde{k}_{0}^{2}} \mathrm{e}^{-\frac{1}{1+4 \tilde{t}^{2}}\left[\tilde{\chi}^{2}-\frac{\tilde{k}_{0}^{2}}{4}-4 \tilde{x} \tilde{x}_{0}\right.}\right] \frac{i}{\mathrm{e}^{1+4 \tilde{t}^{2}}\left[2 \tilde{x}_{0}+2 \tilde{x}^{2}-\tilde{t} \frac{\tilde{k}_{0}^{2}}{2}\right]} \sqrt{1-2 i \tilde{t}} .
\end{aligned}
$$

Using the De Moivre formula (1707) and geometric relations [15]) we obtain

$$
\left.\psi(\tilde{x}, \tilde{t})=\sqrt[4]{\frac{2}{\pi\left(1+4 \tilde{t}^{2}\right)}} \mathrm{e}^{-\frac{1}{4} \tilde{k}_{0}^{2}} \mathrm{e}^{-\frac{1}{1+4 \tilde{t}^{2}}\left[\tilde{x}^{2}-\frac{\tilde{k}_{0}^{2}}{4}-4 \tilde{x} \tilde{k}_{0}\right.}\right] \mathrm{e}^{i\left\{\frac{1}{1+4 \tilde{t}^{2}}\left[2 \tilde{k}_{0}+2 \tilde{x}^{2}-\tilde{t} \frac{\tilde{k}_{0}^{2}}{2}\right]+0.5 \arctan (-2 \tilde{t})\right\}}
$$

Then

$$
\psi \cdot(\tilde{x}, \tilde{t})=\sqrt[4]{\frac{2}{\pi\left(1+4 \tilde{t}^{2}\right)}} \mathrm{e}^{-\frac{1}{4} \tilde{k}_{0}^{2}} \mathrm{e}^{-\frac{1}{1+4 \tilde{t}^{2}}\left[\tilde{x}^{2}-\frac{\tilde{k}_{0}^{2}}{4}-4 \tilde{x} \tilde{x}_{0}\right]} \mathrm{e}^{-i\left\{\frac{1}{1+4 \tilde{t}^{2}}\left[2 \tilde{x} \tilde{x}_{0}+2 \tilde{\tilde{x}} \tilde{x}^{2}-\hat{t} \frac{\tilde{E}_{0}^{2}}{2}\right]+0.5 \arctan (-2 \tilde{t})\right\}}
$$

Now we can calculate

$$
\left.|\psi(\tilde{x}, \tilde{t})|^{2}=\psi \psi^{*}=\sqrt{\frac{2}{\pi\left(1+4 \tilde{t}^{2}\right)}} \mathrm{e}^{-\frac{1}{2} \tilde{k}_{0}^{2}} \mathrm{e}^{-\frac{2}{1+4 \tilde{t}^{2}}\left\{\tilde{x}^{2}-\frac{\tilde{k}_{0}^{2}}{4}-4 \tilde{k}_{0} \tilde{x}\right.}\right\} .
$$


As we see $|\psi(\tilde{x}, \tilde{t})|^{2} \rightarrow 0$ if $\tilde{t} \rightarrow \infty$. Really, the dispersive behavior of this wave packet is obvious by looking at the probability density (1.33). For the large $\tilde{t}$

$$
\rho=|\psi(\tilde{x}, \tilde{t})|^{2}=\frac{1}{\tilde{t}} \frac{1}{\sqrt{2 \pi}} \mathrm{e}^{-\frac{1}{2} \tilde{k}_{0}^{2}} \mathrm{e}^{-\frac{1}{2 \tilde{t}^{2}}\left\{\tilde{x}^{2}-\frac{\tilde{k}_{0}^{2}}{4}-4 \tilde{k_{0}} \tilde{x}\right\}} \sim \frac{1}{\tilde{t}} \mathrm{e}^{\frac{2}{\tilde{t}} \tilde{k}_{0} \tilde{x}} \sim \frac{1}{\tilde{t}^{2}} .
$$

It is evident that this dispersive wave packet, while moving with constant group velocity $\left(\tilde{k}_{0}=\right.$ const $)$, is delocalizing rapidly (if $\tilde{t} \rightarrow \infty$ ). This fact leads to dramatic consequences not only for the theoretical physics but for biology also (see [16]).

The nonlocal physics radically improve the situation; as a result we obtain stable solitons [2] [3] [4] [5] [17].

Let us consider the $\beta$ dimension, using (1.20).

$$
[\mathbf{v}] \frac{\mathrm{cm}}{\mathrm{s}}=\left[\frac{\hbar}{\mathrm{m}} \frac{\partial \beta}{\partial \mathbf{r}}\right] \frac{\mathrm{erg} \cdot \mathrm{s}}{\mathrm{g} \cdot \mathrm{cm}}=\frac{\mathrm{g} \cdot \mathrm{cm}^{2}}{\mathrm{~s}^{2}} \frac{\mathrm{s}}{\mathrm{g} \cdot \mathrm{cm}} \beta=\frac{\mathrm{cm}}{\mathrm{s}} \beta .
$$

It means that $\beta$ is a dimensionless value. The abstract of the classic Madelung's article [11] contains only one brilliant phrase: "It is shown that the Schrödinger equation for one-electron problems can be transformed into the form of hydrodynamic equations".

This means that a single electron can be "smeared" into hydrodynamics!

It is not surprising that the generalized hydrodynamic theory also works for large Knudsen numbers. SE is transformed into the hydrodynamic form of Madelung without additional assumptions. Numerical methods of hydrodynamics are well developed. As a result, back in the late seventies of the last century, we implemented systematic calculations [2] of quantum mechanics problems using the equations of quantum hydrodynamics.

However, practically the hydrodynamics of Madelung was forgotten. There was a (erroneous) opinion that quantization could not be introduced into Madelung hydrodynamics. It is interesting to note that in the famous book by David Bohm (Quantum Theory, New York, Prentice-Hall, Inc., 1952), containing 728 pages in Russian translation, Madelung's name is not even mentioned!

$\mathrm{SE}$ is reduced to a system of equations consisting of the continuity equation and a special case of the motion equation with an additional quantum potential $U_{q u}$ proportional to $\hbar^{2}$. The physical meaning and origin of the quantum potential is discussed in [17] [18]. The SE is obtained within the framework of the classical theory of complex variables and cannot contain an independent energy equation in principle. As a result, a palliative approach is used in many cases, when the solution of dissipative problems of quantum mechanics is found by formally introducing $U_{q u}$ into classical hydrodynamic equations.

Despite the non-dissipative form of the equations in the form of Euler equations, the Schrödinger equation becomes reversible with respect to the replacement of the time direction $t \rightarrow-t$ only after the transition to complex conjugate quantities in the SE. In other words, the "derivation" of the SE using the 
wave function in the form $\psi=\mathrm{e}^{i(\omega t-k x)}$ leads to another system of hydrodynamic equations:

$$
\begin{gathered}
-\frac{\partial \rho}{\partial t}+\frac{\partial}{\partial \mathbf{r}} \cdot(\rho \mathbf{v})=0, \\
-\frac{\partial \mathbf{v}}{\partial t}+\left(\mathbf{v} \cdot \frac{\partial}{\partial \mathbf{r}}\right) \mathbf{v}=-\frac{1}{m} \frac{\partial}{\partial \mathbf{r}} U^{\bullet},
\end{gathered}
$$

This means that the generalized hydrodynamic equations (GHE) contain an implicit approximation against the direction of the arrow of time. The theory of irreversible processes denies the possibility of the existence of such processes. However, the Poincare-Zermelo theorem allows, in principle, the return of the system to its original state if the evolution of the system obeys the dynamics of Newton.

The next question of fundamental importance-is it possible to obtain the Schrödinger equation from the local Liouville equation, namely from the Liouville equation written with respect to a single-particle distribution function $f(x, \mathrm{p}, t)$

$$
\frac{\partial f}{\partial t}+\frac{\mathrm{p}}{m} \frac{\partial f}{\partial x}+F(x) \frac{\partial f}{\partial \mathrm{p}}=0
$$

where $F(x)=-\frac{\partial U}{\partial x}$ is an external force acting on a particle of mass. The answer to this question is no. In essence, Equation (1.38) is a collisionless local Boltzmann equation. It is established [9] [10] that the Liouville equation can be transformed into SE only after the artificial introduction of spatial nonlocality without the introduction of nonlocality in time (E. Carnovalli Jr, H.M. Franca, B.V. Alexeev).

\section{Generalized Hydrodynamic Equations of Nonlocal Physics and Madelung Wave Function}

The generalized hydrodynamic equations (GHE) can be obtained from the nonlocal kinetic equation in the frame of the Enskog procedure, (see for example [2] [3] [4] [5]). Generally speaking to GHE should be added the system of generalized Maxwell equations (for example in the form of the generalized Poisson equation for electric potential) and gravitational equations (for example in the form of the generalized Poisson equation for gravitational potential). For example

$$
\frac{\partial}{\partial \mathbf{r}} \cdot \mathbf{F}^{(1)}=-4 \pi \gamma_{N}\left[\rho-\tau\left(\frac{\partial \rho}{\partial t}+\frac{\partial}{\partial \mathbf{r}} \cdot \rho \mathbf{v}_{0}\right)\right]
$$

(Continuity equation for species $\alpha$ )

$$
\begin{aligned}
& \frac{\partial}{\partial t}\left\{\rho_{\alpha}-\tau_{\alpha}\left[\frac{\partial \rho_{\alpha}}{\partial t}+\frac{\partial}{\partial \mathbf{r}} \cdot\left(\rho_{\alpha} \mathbf{v}_{0}\right)\right]\right\}+\frac{\partial}{\partial \mathbf{r}} \cdot\left\{\rho_{\alpha} \mathbf{v}_{0}-\tau_{\alpha}\left[\frac{\partial}{\partial t}\left(\rho_{\alpha} \mathbf{v}_{0}\right)\right.\right. \\
& \left.\left.+\frac{\partial}{\partial \mathbf{r}} \cdot\left(\rho_{\alpha} \mathbf{v}_{0} \mathbf{v}_{0}\right)+\overrightarrow{\mathrm{I}} \cdot \frac{\partial p_{\alpha}}{\partial \mathbf{r}}-\rho_{\alpha} \mathbf{F}_{\alpha}^{(1)}-\frac{q_{\alpha}}{m_{\alpha}} \rho_{\alpha} \mathbf{v}_{0} \times \mathbf{B}\right]\right\}=R_{\alpha} .
\end{aligned}
$$


(Continuity equation for mixture)

$$
\begin{aligned}
& \frac{\partial}{\partial t}\left\{\rho-\sum_{\alpha} \tau_{\alpha}\left[\frac{\partial \rho_{\alpha}}{\partial t}+\frac{\partial}{\partial \mathbf{r}} \cdot\left(\rho_{\alpha} \mathbf{v}_{0}\right)\right]\right\}+\frac{\partial}{\partial \mathbf{r}} \cdot\left\{\rho \mathbf{v}_{0}-\sum_{\alpha} \tau_{\alpha}\left[\frac{\partial}{\partial t}\left(\rho_{\alpha} \mathbf{v}_{0}\right)\right.\right. \\
& \left.\left.+\frac{\partial}{\partial \mathbf{r}} \cdot\left(\rho_{\alpha} \mathbf{v}_{0} \mathbf{v}_{0}\right)+\overrightarrow{\mathrm{I}} \cdot \frac{\partial p_{\alpha}}{\partial \mathbf{r}}-\rho_{\alpha} \mathbf{F}_{\alpha}^{(1)}-\frac{q_{\alpha}}{m_{\alpha}} \rho_{\alpha} \mathbf{v}_{0} \times \mathbf{B}\right]\right\}=0 .
\end{aligned}
$$

(Momentum equation for species $\alpha$ )

$$
\begin{aligned}
& \frac{\partial}{\partial t}\left\{\rho_{\alpha} \mathbf{v}_{0}-\tau_{\alpha}\left[\frac{\partial}{\partial t}\left(\rho_{\alpha} \mathbf{v}_{0}\right)+\frac{\partial}{\partial \mathbf{r}} \cdot \rho_{\alpha} \mathbf{v}_{0} \mathbf{v}_{0}+\frac{\partial p_{\alpha}}{\partial \mathbf{r}}-\rho_{\alpha} \mathbf{F}_{\alpha}^{(1)}-\frac{q_{\alpha}}{m_{\alpha}} \rho_{\alpha} \mathbf{v}_{0} \times \mathbf{B}\right]\right\} \\
& -\mathbf{F}_{\alpha}^{(1)}\left[\rho_{\alpha}-\tau_{\alpha}\left(\frac{\partial \rho_{\alpha}}{\partial t}+\frac{\partial}{\partial \mathbf{r}} \cdot\left(\rho_{\alpha} \mathbf{v}_{0}\right)\right)\right]-\frac{q_{\alpha}}{m_{\alpha}}\left\{\rho_{\alpha} \mathbf{v}_{0}-\tau_{\alpha}\left[\frac{\partial}{\partial t}\left(\rho_{\alpha} \mathbf{v}_{0}\right)\right.\right. \\
& \left.\left.+\frac{\partial}{\partial \mathbf{r}} \cdot \rho_{\alpha} \mathbf{v}_{0} \mathbf{v}_{0}+\frac{\partial p_{\alpha}}{\partial \mathbf{r}}-\rho_{\alpha} \mathbf{F}_{\alpha}^{(1)}-\frac{q_{\alpha}}{m_{\alpha}} \rho_{\alpha} \mathbf{v}_{0} \times \mathbf{B}\right]\right\} \times \mathbf{B}+\frac{\partial}{\partial \mathbf{r}} \cdot\left\{\rho_{\alpha} \mathbf{v}_{0} \mathbf{v}_{0}+p_{\alpha} \overrightarrow{\mathrm{I}}\right. \\
& -\tau_{\alpha}\left[\frac{\partial}{\partial t}\left(\rho_{\alpha} \mathbf{v}_{0} \mathbf{v}_{0}+p_{\alpha} \tilde{\mathrm{I}}\right)+\frac{\partial}{\partial \mathbf{r}} \cdot \rho_{\alpha}\left(\mathbf{v}_{0} \mathbf{v}_{0}\right) \mathbf{v}_{0}+2 \overrightarrow{\mathrm{I}}\left(\frac{\partial}{\partial \mathbf{r}} \cdot\left(p_{\alpha} \mathbf{v}_{0}\right)\right)+\frac{\partial}{\partial \mathbf{r}} \cdot\left(\overrightarrow{\mathrm{I}} p_{\alpha} \mathbf{v}_{0}\right)\right. \\
& \left.\left.-\mathbf{F}_{\alpha}^{(1)} \rho_{\alpha} \mathbf{v}_{0}-\rho_{\alpha} \mathbf{v}_{0} \mathbf{F}_{\alpha}^{(1)}-\frac{q_{\alpha}}{m_{\alpha}} \rho_{\alpha}\left[\mathbf{v}_{0} \times \mathbf{B}\right] \mathbf{v}_{0}-\frac{q_{\alpha}}{m_{\alpha}} \rho_{\alpha} \mathbf{v}_{0}\left[\mathbf{v}_{0} \times \mathbf{B}\right]\right]\right\} \\
& =\int m_{\alpha} \mathbf{v}_{\alpha} J_{\alpha}^{s t, e l} \mathrm{~d} \mathbf{v}_{\alpha}+\int m_{\alpha} \mathbf{v}_{\alpha} J_{\alpha}^{\text {st,inel }} \mathrm{d} \mathbf{v}_{\alpha} .
\end{aligned}
$$

(Momentum equation for mixture)

$$
\begin{aligned}
& \frac{\partial}{\partial t}\left\{\rho \mathbf{v}_{0}-\sum_{\alpha} \tau_{\alpha}\left[\frac{\partial}{\partial t}\left(\rho_{\alpha} \mathbf{v}_{0}\right)+\frac{\partial}{\partial \mathbf{r}} \cdot \rho_{\alpha} \mathbf{v}_{0} \mathbf{v}_{0}+\frac{\partial p_{\alpha}}{\partial \mathbf{r}}-\rho_{\alpha} \mathbf{F}_{\alpha}^{(1)}-\frac{q_{\alpha}}{m_{\alpha}} \rho_{\alpha} \mathbf{v}_{0} \times \mathbf{B}\right]\right\} \\
& -\sum_{\alpha} \mathbf{F}_{\alpha}^{(1)}\left[\rho_{\alpha}-\tau_{\alpha}\left(\frac{\partial \rho_{\alpha}}{\partial t}+\frac{\partial}{\partial \mathbf{r}} \cdot\left(\rho_{\alpha} \mathbf{v}_{0}\right)\right)\right]-\sum_{\alpha} \frac{q_{\alpha}}{m_{\alpha}}\left\{\rho_{\alpha} \mathbf{v}_{0}-\tau_{\alpha}\left[\frac{\partial}{\partial t}\left(\rho_{\alpha} \mathbf{v}_{0}\right)\right.\right. \\
& \left.\left.+\frac{\partial}{\partial \mathbf{r}} \cdot \rho_{\alpha} \mathbf{v}_{0} \mathbf{v}_{0}+\frac{\partial p_{\alpha}}{\partial \mathbf{r}}-\rho_{\alpha} \mathbf{F}_{\alpha}^{(1)}-\frac{q_{\alpha}}{m_{\alpha}} \rho_{\alpha} \mathbf{v}_{0} \times \mathbf{B}\right]\right\} \times \mathbf{B}+\frac{\partial}{\partial \mathbf{r}} \cdot\left\{\rho \mathbf{v}_{0} \mathbf{v}_{0}+p \overrightarrow{\mathrm{I}}\right. \\
& -\sum_{\alpha} \tau_{\alpha}\left[\frac{\partial}{\partial t}\left(\rho_{\alpha} \mathbf{v}_{0} \mathbf{v}_{0}+p_{\alpha} \overrightarrow{\mathrm{I}}\right)+\frac{\partial}{\partial \mathbf{r}} \cdot \rho_{\alpha}\left(\mathbf{v}_{0} \mathbf{v}_{0}\right) \mathbf{v}_{0}+2 \overrightarrow{\mathrm{I}}\left(\frac{\partial}{\partial \mathbf{r}} \cdot\left(p_{\alpha} \mathbf{v}_{0}\right)\right)+\frac{\partial}{\partial \mathbf{r}} \cdot\left(\overrightarrow{\mathrm{I}} p_{\alpha} \mathbf{v}_{0}\right)\right. \\
& \left.\left.-\mathbf{F}_{\alpha}^{(1)} \rho_{\alpha} \mathbf{v}_{0}-\rho_{\alpha} \mathbf{v}_{0} \mathbf{F}_{\alpha}^{(1)}-\frac{q_{\alpha}}{m_{\alpha}} \rho_{\alpha}\left[\mathbf{v}_{0} \times \mathbf{B}\right] \mathbf{v}_{0}-\frac{q_{\alpha}}{m_{\alpha}} \rho_{\alpha} \mathbf{v}_{0}\left[\mathbf{v}_{0} \times \mathbf{B}\right]\right]\right\}=0 .
\end{aligned}
$$

(Energy equation for $\alpha$ species)

$$
\begin{aligned}
& \frac{\partial}{\partial t}\left\{\frac{\rho_{\alpha} v_{0}^{2}}{2}+\frac{3}{2} p_{\alpha}+\varepsilon_{\alpha} n_{\alpha}-\tau_{\alpha}\left[\frac{\partial}{\partial t}\left(\frac{\rho_{\alpha} v_{0}^{2}}{2}+\frac{3}{2} p_{\alpha}+\varepsilon_{\alpha} n_{\alpha}\right)+\frac{\partial}{\partial \mathbf{r}} \cdot\left(\frac{1}{2} \rho_{\alpha} v_{0}^{2} \mathbf{v}_{0}\right.\right.\right. \\
& \left.\left.\left.+\frac{5}{2} p_{\alpha} \mathbf{v}_{0}+\varepsilon_{\alpha} n_{\alpha} \mathbf{v}_{0}\right)-\mathbf{F}_{\alpha}^{(1)} \cdot \rho_{\alpha} \mathbf{v}_{0}\right]\right\}+\frac{\partial}{\partial \mathbf{r}} \cdot\left\{\frac{1}{2} \rho_{\alpha} v_{0}^{2} \mathbf{v}_{0}+\frac{5}{2} p_{\alpha} \mathbf{v}_{0}+\varepsilon_{\alpha} n_{\alpha} \mathbf{v}_{0}\right. \\
& -\tau_{\alpha}\left[\frac{\partial}{\partial t}\left(\frac{1}{2} \rho_{\alpha} v_{0}^{2} \mathbf{v}_{0}+\frac{5}{2} p_{\alpha} \mathbf{v}_{0}+\varepsilon_{\alpha} n_{\alpha} \mathbf{v}_{0}\right)+\frac{\partial}{\partial \mathbf{r}} \cdot\left(\frac{1}{2} \rho_{\alpha} v_{0}^{2} \mathbf{v}_{0} \mathbf{v}_{0}+\frac{7}{2} p_{\alpha} \mathbf{v}_{0} \mathbf{v}_{0}\right.\right. \\
& \left.+\frac{1}{2} p_{\alpha} v_{0}^{2} \overrightarrow{\mathrm{I}}+\frac{5}{2} \frac{p_{\alpha}^{2}}{\rho_{\alpha}} \overrightarrow{\mathrm{I}}+\varepsilon_{\alpha} n_{\alpha} \mathbf{v}_{0} \mathbf{v}_{0}+\varepsilon_{\alpha} \frac{p_{\alpha}}{m_{\alpha}} \overrightarrow{\mathrm{I}}\right)-\rho_{\alpha} \mathbf{F}_{\alpha}^{(1)} \cdot \mathbf{v}_{0} \mathbf{v}_{0}-p_{\alpha} \mathbf{F}_{\alpha}^{(1)} \cdot \overrightarrow{\mathrm{I}}
\end{aligned}
$$




$$
\begin{aligned}
& -\frac{1}{2} \rho_{\alpha} v_{0}^{2} \mathbf{F}_{\alpha}^{(1)}-\frac{3}{2} \mathbf{F}_{\alpha}^{(1)} p_{\alpha}-\frac{\rho_{\alpha} v_{0}^{2}}{2} \frac{q_{\alpha}}{m_{\alpha}}\left[\mathbf{v}_{0} \times \mathbf{B}\right]-\frac{5}{2} p_{\alpha} \frac{q_{\alpha}}{m_{\alpha}}\left[\mathbf{v}_{0} \times \mathbf{B}\right] \\
& \left.\left.-\varepsilon_{\alpha} n_{\alpha} \frac{q_{\alpha}}{m_{\alpha}}\left[\mathbf{v}_{0} \times \mathbf{B}\right]-\varepsilon_{\alpha} n_{\alpha} \mathbf{F}_{\alpha}^{(1)}\right]\right\}-\left\{\rho_{\alpha} \mathbf{F}_{\alpha}^{(1)} \cdot \mathbf{v}_{0}-\tau_{\alpha}\left[\mathbf { F } _ { \alpha } ^ { ( 1 ) } \cdot \left(\frac{\partial}{\partial t}\left(\rho_{\alpha} \mathbf{v}_{0}\right)\right.\right.\right. \\
& \left.\left.\left.+\frac{\partial}{\partial \mathbf{r}} \cdot \rho_{\alpha} \mathbf{v}_{0} \mathbf{v}_{0}+\frac{\partial}{\partial \mathbf{r}} \cdot p_{\alpha} \vec{I}-\rho_{\alpha} \mathbf{F}_{\alpha}^{(1)}-q_{\alpha} n_{\alpha}\left[\mathbf{v}_{0} \times \mathbf{B}\right]\right)\right]\right\} \\
& =\int\left(\frac{m_{\alpha} v_{\alpha}^{2}}{2}+\varepsilon_{\alpha}\right) J_{\alpha}^{s t, e l} \mathrm{~d} \mathbf{v}_{\alpha}+\int\left(\frac{m_{\alpha} v_{\alpha}^{2}}{2}+\varepsilon_{\alpha}\right) J_{\alpha}^{s t, i n e l} \mathrm{~d} \mathbf{v}_{\alpha} .
\end{aligned}
$$

(Energy equation for mixture)

$$
\begin{aligned}
& \frac{\partial}{\partial t}\left\{\frac{\rho v_{0}^{2}}{2}+\frac{3}{2} p+\sum_{\alpha} \varepsilon_{\alpha} n_{\alpha}-\sum_{\alpha} \tau_{\alpha}\left[\frac{\partial}{\partial t}\left(\frac{\rho_{\alpha} v_{0}^{2}}{2}+\frac{3}{2} p_{\alpha}+\varepsilon_{\alpha} n_{\alpha}\right)+\frac{\partial}{\partial \mathbf{r}} \cdot\left(\frac{1}{2} \rho_{\alpha} v_{0}^{2} \mathbf{v}_{0}\right.\right.\right. \\
& \left.\left.\left.+\frac{5}{2} p_{\alpha} \mathbf{v}_{0}+\varepsilon_{\alpha} n_{\alpha} \mathbf{v}_{0}\right)-\mathbf{F}_{\alpha}^{(1)} \cdot \rho_{\alpha} \mathbf{v}_{0}\right]\right\}+\frac{\partial}{\partial \mathbf{r}} \cdot\left\{\frac{1}{2} \rho v_{0}^{2} \mathbf{v}_{0}+\frac{5}{2} p \mathbf{v}_{0}+\mathbf{v}_{0} \sum \varepsilon_{\alpha} \varepsilon_{\alpha}\right. \\
& -\sum_{\alpha} \tau_{\alpha}\left[\frac{\partial}{\partial t}\left(\frac{1}{2} \rho_{\alpha} v_{0}^{2} \mathbf{v}_{0}+\frac{5}{2} p_{\alpha} \mathbf{v}_{0}+\varepsilon_{\alpha} n_{\alpha} \mathbf{v}_{0}\right)+\frac{\partial}{\partial \mathbf{r}} \cdot\left(\frac{1}{2} \rho_{\alpha} v_{0}^{2} \mathbf{v}_{0} \mathbf{v}_{0}+\frac{7}{2} p_{\alpha} \mathbf{v}_{0} \mathbf{v}_{0}\right.\right. \\
& \left.+\frac{1}{2} p_{\alpha} v_{0}^{2} \overrightarrow{\mathrm{I}}+\frac{5}{2} \frac{p_{\alpha}^{2}}{\rho_{\alpha}} \overrightarrow{\mathrm{I}}+\varepsilon_{\alpha} n_{\alpha} \mathbf{v}_{0} \mathbf{v}_{0}+\varepsilon_{\alpha} \frac{p_{\alpha}}{m_{\alpha}} \overrightarrow{\mathrm{I}}\right)-\rho_{\alpha} \mathbf{F}_{\alpha}^{(1)} \cdot \mathbf{v}_{0} \mathbf{v}_{0}-p_{\alpha} \mathbf{F}_{\alpha}^{(1)} \cdot \overrightarrow{\mathrm{I}} \\
& -\frac{1}{2} \rho_{\alpha} v_{0}^{2} \mathbf{F}_{\alpha}^{(1)}-\frac{3}{2} \mathbf{F}_{\alpha}^{(1)} p_{\alpha}-\frac{\rho_{\alpha} v_{0}^{2}}{2} \frac{q_{\alpha}}{m_{\alpha}}\left[\mathbf{v}_{0} \times \mathbf{B}\right]-\frac{5}{2} p_{\alpha} \frac{q_{\alpha}}{m_{\alpha}}\left[\mathbf{v}_{0} \times \mathbf{B}\right] \\
& \left.\left.-\varepsilon_{\alpha} n_{\alpha} \frac{q_{\alpha}}{m_{\alpha}}\left[\mathbf{v}_{0} \times \mathbf{B}\right]-\varepsilon_{\alpha} n_{\alpha} \mathbf{F}_{\alpha}^{(1)}\right]\right\}-\left\{\mathbf{v}_{0} \cdot \sum_{\alpha} \rho_{\alpha} \mathbf{F}_{\alpha}^{(1)}-\sum_{\alpha} \tau_{\alpha}\left[\mathbf { F } _ { \alpha } ^ { ( 1 ) } \cdot \left(\frac{\partial}{\partial t}\left(\rho_{\alpha} \mathbf{v}_{0}\right)(2.7)\right.\right.\right. \\
& \left.\left.\left.+\frac{\partial}{\partial \mathbf{r}} \cdot \rho_{\alpha} \mathbf{v}_{0} \mathbf{v}_{0}+\frac{\partial}{\partial \mathbf{r}} \cdot p_{\alpha} \overrightarrow{\mathrm{I}}-\rho_{\alpha} \mathbf{F}_{\alpha}^{(1)}-q_{\alpha} n_{\alpha}\left[\mathbf{v}_{0} \times \mathbf{B}\right]\right)\right]\right\}=0 .
\end{aligned}
$$

Here $\mathbf{F}_{\alpha}^{(1)}$ are the forces of the non-magnetic origin acting on the mass unit, B -magnetic induction, $\overrightarrow{\mathrm{I}}$-unit tensor, $q_{\alpha}$-charge of the $\alpha$-component particle, $p_{\alpha}$-static pressure for $\alpha$-component, $\varepsilon_{\alpha}$-internal energy for the particles of $\alpha$-component, $\rho_{\alpha}$ is density for $\alpha$ species, $\mathbf{v}_{0}$-hydrodynamic velocity for mixture, $\tau_{\alpha}$-non-local parameter.

For many decades (due to natural historical reasons), the equations of quantum mechanics were written in terms of the wave function $\psi$. Is such a notation possible for nonlinear Equations (2.1)-(2.7)?

This procedure is quite possible if we take into account that the hydrodynamic correspondence of the wave function and the variables of quantum hydrodynamics is introduced using the Madelung relations:

$$
\begin{aligned}
\psi(x, y, z, t) & =\alpha(x, y, z, t) \mathrm{e}^{i \beta(x, y, z, t)}, \\
\rho & =\alpha^{2}, \\
\mathbf{v}_{0} & =\frac{\hbar}{m} \frac{\partial \beta}{\partial \mathbf{r}}
\end{aligned}
$$

Of course, quantum pressure $p$ cannot be represented through a wave func- 
tion, since Schrödinger-Madelung quantum mechanics does not contain an independent equation of energy (and, therefore, pressure $p$ ).

Thus, at the first glance, the nonlocal hydrodynamic equations together with the Madelung conditions (2.8)-(2.10) exhaust the problem.

\section{About the Non-Local Hydrodynamic Equations in $\psi$-Interpretation}

The following conclusions of fundamental importance can be made:

1) Madelung's quantum hydrodynamics is equivalent to the Schrödinger equation and leads to a description of the evolution of a quantum particle in the form of the Euler motion equation and the continuity equation. We have a consequence of the nonlocal Liouville equation as a result of the local spatial approximation of the nonlocal equations.

2) Generalized Boltzmann physical kinetics contains strict consideration of nonlocal effects in space and time and, after a limiting transition to the local approximation, leads to a nonlocality parameter $\tau_{q u}$, which at the quantum level is consistent with the Heisenberg uncertainty principle.

3) Generalized hydrodynamic equations (GHE) contain SE as a deep special case. In other words, we have formulated explicitly [9] [10] all the assumptions that must be implemented to obtain SE from GHE. At the final stage, for simplicity, a non-stationary 1D Madelung-Schrodinger model was obtained without taking into account external forces. So, we can state that we have a deep special case of generalized hydrodynamic equations. This means that a new quantum mechanics of dissipative processes has been created.

4) The Boltzmann equation fundamentally "does not work" at distances of the order of the radius of interaction of particles and, therefore, cannot be effectively used in the theoretical study of nanotechnology problems even within the framework of "plausible" models.

GHE have the extremely important special cases for astrophysics, when the density $\rho \rightarrow 0$ (the initial stage of the evolution of the Universe, the Big Bang, see for example [4] [19]) and when the density $\rho \rightarrow \infty$ (the evolution of a black hole, [4] [20]. Both limiting cases have neither physical nor mathematical meaning in "classical" hydrodynamics. Thus, we have a unified statistical theory of dissipative structures having a hydrodynamic form.

Between these global (and rather new problems) we have a very old problem about the physical sense of wave function and quantum jumps. When surveying the literature, one often gets the impression that Schrödinger held several distinct interpretations of quantum mechanics, and practically in all cases he denied the interpretation of the Copenhagen group. It is known the most impressive Schrödinger reaction: "If we have to go on with these damned quantum jumps, then I'm sorry that I ever got involved".

In NLQH we needn't to use the formalism of the $\psi$ function description. Then we needn't finding the physical interpretation of $\psi$ function. 
From position of nonlocal physics $\psi$-function is only a mathematical function (between many others) defining by relations (2.8)-(2.10). The attempt to find any physical sense for $\psi$ has no reason.

But physical community accustomed to "the $\psi$ interpretation". Then it is reasonable to find "the $\psi$ interpretation" of non-local hydrodynamic equations from the simplest considerations avoiding complex mathematical formalism.

Let us consider the transformation of the nonlocal continuity equation in the equations of the wave quantum mechanics including the Schrödinger-Madelung model. We transform the nonlocal continuity equation written for one species flow corresponding to the Schrödinger-Madelung model (see also (2.3) and (2.8)-(2.10)). For the simplicity we do not take into account the influence of magnetic effects

$$
\frac{\partial}{\partial t}\left\{\rho-\tau_{q u}\left[\frac{\partial \rho}{\partial t}+\frac{\partial}{\partial \mathbf{r}} \cdot(\rho \mathbf{v})\right]\right\}+\frac{\partial}{\partial \mathbf{r}} \cdot\left\{\rho \mathbf{v}-\tau_{q u}\left[\frac{\partial}{\partial t}(\rho \mathbf{v})+\frac{\partial}{\partial \mathbf{r}} \cdot \rho \mathbf{v v}-\rho \mathbf{F}\right]\right\}=0 .
$$

Using the Madelung identifications

$$
\rho=\alpha^{2}=\psi \psi^{*},
$$

and

$$
\mathbf{v}=\frac{\partial}{\partial \mathbf{r}}\left(\frac{\beta \hbar}{m}\right)
$$

we find

$$
\psi=\alpha \mathrm{e}^{i \beta}, \ln \psi=\ln \alpha+i \beta, \quad i \ln \psi=i \ln \alpha-\beta,
$$

or

$$
\beta=i \ln \alpha-i \ln \psi
$$

Then

$$
\frac{\partial \beta}{\partial \mathbf{r}}=i \frac{\partial \ln \sqrt{|\psi|^{2}}}{\partial \mathbf{r}}-i \frac{\partial \ln \psi}{\partial \mathbf{r}},
$$

Let us rewrite the continuity equation

$$
\begin{aligned}
& \frac{\partial}{\partial t}\left\{\alpha^{2}-\tau_{q u}\left[\frac{\partial \alpha^{2}}{\partial t}+\frac{\partial}{\partial \mathbf{r}} \cdot\left(\alpha^{2} \frac{\partial}{\partial \mathbf{r}}\left(\frac{\beta \hbar}{m}\right)\right)\right]\right\}+\frac{\partial}{\partial \mathbf{r}} \cdot\left\{\alpha^{2} \frac{\partial}{\partial \mathbf{r}}\left(\frac{\beta \hbar}{m}\right)\right. \\
& \left.-\tau_{q u}\left\{\frac{\partial}{\partial t}\left[\alpha^{2} \frac{\partial}{\partial \mathbf{r}}\left(\frac{\beta \hbar}{m}\right)\right]+\frac{\partial}{\partial \mathbf{r}} \cdot\left[\alpha^{2} \frac{\partial}{\partial \mathbf{r}}\left(\frac{\beta \hbar}{m}\right) \frac{\partial}{\partial \mathbf{r}}\left(\frac{\beta \hbar}{m}\right)\right]-\alpha^{2} \mathbf{F}\right\}\right\}=0
\end{aligned}
$$

The subsequent transformations are of a fundamentally important nature and we will give detailed calculations

$$
\begin{aligned}
& \frac{\partial}{\partial t}\left\{\alpha^{2}-\tau_{q u}\left[\frac{\partial \alpha^{2}}{\partial t}+\frac{\hbar}{m} \frac{\partial}{\partial \mathbf{r}} \cdot\left(\alpha^{2} \frac{\partial \beta}{\partial \mathbf{r}}\right)\right]\right\} \\
& +\frac{\partial}{\partial \mathbf{r}} \cdot\left\{\alpha^{2} \frac{\hbar}{m} \frac{\partial \beta}{\partial \mathbf{r}}-\tau_{q u}\left\{\frac{\partial}{\partial t}\left[\alpha^{2} \frac{\hbar}{m} \frac{\partial \beta}{\partial \mathbf{r}}\right]+\frac{\partial}{\partial \mathbf{r}} \cdot\left[\alpha^{2}\left(\frac{\hbar}{m}\right)^{2} \frac{\partial \beta}{\partial \mathbf{r}} \frac{\partial \beta}{\partial \mathbf{r}}\right]-\alpha^{2} \mathbf{F}\right\}\right\}=0
\end{aligned}
$$


or

$$
\begin{aligned}
& \frac{\partial \psi^{2}}{\partial t}-\frac{\partial}{\partial t}\left\{\tau_{q u}\left[\frac{\partial \psi^{2}}{\partial t}+\frac{\hbar}{m} \frac{\partial}{\partial \mathbf{r}} \cdot\left(\psi^{2} \frac{\partial \beta}{\partial \mathbf{r}}\right)\right]\right\} \\
& +\frac{\partial}{\partial \mathbf{r}} \cdot\left\{\psi^{2} \frac{\hbar}{m} \frac{\partial \beta}{\partial \mathbf{r}}-\tau_{q u}\left\{\frac{\partial}{\partial t}\left[\psi^{2} \frac{\hbar}{m} \frac{\partial \beta}{\partial \mathbf{r}}\right]+\frac{\partial}{\partial \mathbf{r}} \cdot\left[\psi^{2}\left(\frac{\hbar}{m}\right)^{2} \frac{\partial \beta}{\partial \mathbf{r}} \frac{\partial \beta}{\partial \mathbf{r}}\right]-\psi^{2} \mathbf{F}\right\}\right\}=0
\end{aligned}
$$

or

$$
\begin{aligned}
& 2 \psi \frac{\partial \psi}{\partial t}-\frac{\partial}{\partial t}\left\{\tau_{q u}\left[\frac{\partial \psi^{2}}{\partial t}+\frac{\hbar}{m} \frac{\partial}{\partial \mathbf{r}} \cdot\left(\psi^{2} \frac{\partial \beta}{\partial \mathbf{r}}\right)\right]\right\}+\psi^{2} \frac{\hbar}{m} \frac{\partial}{\partial \mathbf{r}} \cdot \frac{\partial \beta}{\partial \mathbf{r}}+2 \psi \frac{\hbar}{m} \frac{\partial \beta}{\partial \mathbf{r}} \cdot \frac{\partial \psi}{\partial \mathbf{r}} \\
& -\frac{\partial}{\partial \mathbf{r}} \cdot\left\{\tau_{q u}\left\{\frac{\partial}{\partial t}\left[\psi^{2} \frac{\hbar}{m} \frac{\partial \beta}{\partial \mathbf{r}}\right]+\frac{\partial}{\partial \mathbf{r}} \cdot\left[\psi^{2}\left(\frac{\hbar}{m}\right)^{2} \frac{\partial \beta}{\partial \mathbf{r}} \frac{\partial \beta}{\partial \mathbf{r}}\right]-\psi^{2} \mathbf{F}\right\}\right\}=0
\end{aligned}
$$

or

$$
\begin{aligned}
& i \hbar \frac{\partial \psi}{\partial t}-\frac{1}{2 \psi} i \hbar \frac{\partial}{\partial t}\left\{\tau_{q u}\left[\frac{\partial \psi^{2}}{\partial t}+\frac{\hbar}{m} \frac{\partial}{\partial \mathbf{r}} \cdot\left(\psi^{2} \frac{\partial \beta}{\partial \mathbf{r}}\right)\right]\right\} \\
& +\psi i \hbar \frac{\hbar}{2 m} \frac{\partial}{\partial \mathbf{r}} \cdot \frac{\partial \beta}{\partial \mathbf{r}}+i \hbar \frac{\hbar}{m} \frac{\partial \beta}{\partial \mathbf{r}} \cdot \frac{\partial \psi}{\partial \mathbf{r}} \\
& -\frac{i \hbar}{2 \psi} \frac{\partial}{\partial \mathbf{r}} \cdot\left\{\tau_{q u}\left\{\frac{\partial}{\partial t}\left[\psi^{2} \frac{\hbar}{m} \frac{\partial \beta}{\partial \mathbf{r}}\right]+\frac{\partial}{\partial \mathbf{r}} \cdot\left[\psi^{2}\left(\frac{\hbar}{m}\right)^{2} \frac{\partial \beta}{\partial \mathbf{r}} \frac{\partial \beta}{\partial \mathbf{r}}\right]-\psi^{2} \mathbf{F}\right\}\right\}=0
\end{aligned}
$$

or

$$
\begin{aligned}
& i \hbar \frac{\partial \psi}{\partial t}-\frac{1}{\psi} i \hbar \frac{\partial}{\partial t}\left\{\tau_{q u} \psi \frac{\partial \psi}{\partial t}\right\}-i \frac{1}{\psi} \frac{\hbar^{2}}{2 m} \frac{\partial}{\partial t}\left\{\tau_{q u} \frac{\partial}{\partial \mathbf{r}} \cdot\left(\psi^{2} \frac{\partial \beta}{\partial \mathbf{r}}\right)\right\} \\
& -\frac{i}{\psi} \frac{\hbar^{2}}{2 m} \frac{\partial}{\partial \mathbf{r}} \cdot\left\{\tau_{q u} \frac{\partial}{\partial t}\left[\psi^{2} \frac{\partial \beta}{\partial \mathbf{r}}\right]\right\} \\
& =\psi \frac{\hbar^{2}}{2 m} \frac{\partial}{\partial \mathbf{r}} \cdot \frac{\partial \ln \sqrt{|\psi|^{2}}}{\partial \mathbf{r}}-\psi \frac{\hbar^{2}}{2 m} \frac{\partial}{\partial \mathbf{r}} \cdot \frac{\partial \ln \psi}{\partial \mathbf{r}}+\frac{\hbar^{2}}{m}\left[\frac{\partial \ln \sqrt{|\psi|^{2}}}{\partial \mathbf{r}}-\frac{\partial \ln \psi}{\partial \mathbf{r}}\right] \cdot \frac{\partial \psi}{\partial \mathbf{r}} \\
& +\frac{i}{\psi} \frac{\hbar^{3}}{2 m^{2}} \frac{\partial}{\partial \mathbf{r}} \cdot\left\{\tau_{q u} \frac{\partial}{\partial \mathbf{r}} \cdot\left[\psi^{2} \frac{\partial \beta}{\partial \mathbf{r}} \frac{\partial \beta}{\partial \mathbf{r}}\right]\right\}-\frac{i}{\psi} \frac{\hbar}{2} \frac{\partial}{\partial \mathbf{r}} \cdot\left\{\tau_{q u} \psi^{2} \mathbf{F}\right\}
\end{aligned}
$$

or

$$
\begin{aligned}
& i \hbar \frac{\partial \psi}{\partial t}-\frac{1}{\psi} i \hbar \frac{\partial}{\partial t}\left\{\tau_{q u} \psi \frac{\partial \psi}{\partial t}\right\}-i \frac{1}{\psi} \frac{\hbar^{2}}{2 m} \frac{\partial}{\partial t}\left\{\tau_{q u} \frac{\partial}{\partial \mathbf{r}} \cdot\left(\psi^{2} \frac{\partial \beta}{\partial \mathbf{r}}\right)\right\} \\
& -\frac{i}{\psi} \frac{\hbar^{2}}{2 m} \frac{\partial}{\partial \mathbf{r}} \cdot\left\{\tau_{q u} \frac{\partial}{\partial t}\left[\psi^{2} \frac{\partial \beta}{\partial \mathbf{r}}\right]\right\} \\
& =\psi \frac{\hbar^{2}}{2 m} \frac{\partial}{\partial \mathbf{r}} \cdot\left[\frac{1}{\sqrt{|\psi|^{2}}} \frac{\partial \sqrt{|\psi|^{2}}}{\partial \mathbf{r}}\right]-\frac{\hbar^{2}}{2 m} \Delta \psi+\frac{\hbar^{2}}{m} \frac{\partial \ln \sqrt{|\psi|^{2}}}{\partial \mathbf{r}} \cdot \frac{\partial \psi}{\partial \mathbf{r}}-\frac{\hbar^{2}}{2 m} \frac{1}{\psi}\left[\frac{\partial \psi}{\partial \mathbf{r}}\right]^{2} \\
& +\frac{i}{\psi} \frac{\hbar^{3}}{2 m^{2}} \frac{\partial}{\partial \mathbf{r}} \cdot\left\{\tau_{q u} \frac{\partial}{\partial \mathbf{r}} \cdot\left[\psi^{2} \frac{\partial \beta}{\partial \mathbf{r}} \frac{\partial \beta}{\partial \mathbf{r}}\right]\right\}-\frac{i}{\psi} \frac{\hbar}{2} \frac{\partial}{\partial \mathbf{r}} \cdot\left\{\tau_{q u} \psi^{2} \mathbf{F}\right\}
\end{aligned}
$$

or 


$$
\begin{aligned}
& i \hbar \frac{\partial \psi}{\partial t}-\frac{1}{\psi} i \hbar \frac{\partial}{\partial t}\left\{\tau_{q u} \psi \frac{\partial \psi}{\partial t}\right\}-i \frac{1}{\psi} \frac{\hbar^{2}}{2 m} \frac{\partial}{\partial t}\left\{\tau_{q u} \frac{\partial}{\partial \mathbf{r}} \cdot\left(\psi^{2} \frac{\partial \beta}{\partial \mathbf{r}}\right)\right\} \\
& -\frac{i}{\psi} \frac{\hbar^{2}}{2 m} \frac{\partial}{\partial \mathbf{r}} \cdot\left\{\tau_{q u} \frac{\partial}{\partial t}\left[\psi^{2} \frac{\partial \beta}{\partial \mathbf{r}}\right]\right\} \\
& =\psi \frac{\hbar^{2}}{2 m} \frac{1}{\sqrt{|\psi|^{2}}} \frac{\partial}{\partial \mathbf{r}} \cdot\left[\frac{\partial \sqrt{|\psi|^{2}}}{\partial \mathbf{r}}\right]+\psi \frac{\hbar^{2}}{2 m} \frac{\partial \sqrt{|\psi|^{2}}}{\partial \mathbf{r}} \frac{\partial}{\partial \mathbf{r}} \cdot\left[\frac{1}{\sqrt{|\psi|^{2}}}\right] \\
& -\frac{\hbar^{2}}{2 m} \Delta \psi+\frac{\hbar^{2}}{m} \frac{\partial \ln \sqrt{|\psi|^{2}}}{\partial \mathbf{r}} \cdot \frac{\partial \psi}{\partial \mathbf{r}}-\frac{\hbar^{2}}{2 m} \frac{1}{\psi}\left[\frac{\partial \psi}{\partial \mathbf{r}}\right]^{2} \\
& +\frac{i}{\psi} \frac{\hbar^{3}}{2 m^{2}} \frac{\partial}{\partial \mathbf{r}} \cdot\left\{\tau_{q u} \frac{\partial}{\partial \mathbf{r}} \cdot\left[\psi^{2} \frac{\partial \beta}{\partial \mathbf{r}} \frac{\partial \beta}{\partial \mathbf{r}}\right]\right\}-\frac{i}{\psi} \frac{\hbar}{2} \frac{\partial}{\partial \mathbf{r}} \cdot\left\{\tau_{q u} \psi^{2} \mathbf{F}\right\}
\end{aligned}
$$

But

$$
U_{q u}=-\frac{\hbar^{2}}{2 m \sqrt{\rho}} \Delta \sqrt{\rho}
$$

is so called the Bohm's quantum potential, where Laplacian $\Delta$ defined as usual

$$
\Delta=\frac{\partial^{2}}{\partial x^{2}}+\frac{\partial^{2}}{\partial y^{2}}+\frac{\partial^{2}}{\partial z^{2}}=\frac{\partial}{\partial \mathbf{r}} \cdot \frac{\partial}{\partial \mathbf{r}} .
$$

Then

$$
\begin{aligned}
& i \hbar \frac{\partial \psi}{\partial t}-\frac{1}{\psi} i \hbar \frac{\partial}{\partial t}\left\{\tau_{q u} \psi \frac{\partial \psi}{\partial t}\right\}-i \frac{1}{\psi} \frac{\hbar^{2}}{2 m} \frac{\partial}{\partial t}\left\{\tau_{q u} \frac{\partial}{\partial \mathbf{r}} \cdot\left(\psi^{2} \frac{\partial \beta}{\partial \mathbf{r}}\right)\right\} \\
& -\frac{i}{\psi} \frac{\hbar^{2}}{2 m} \frac{\partial}{\partial \mathbf{r}} \cdot\left\{\tau_{q u} \frac{\partial}{\partial t}\left[\psi^{2} \frac{\partial \beta}{\partial \mathbf{r}}\right]\right\} \\
& =-\psi U_{q u}+\psi \frac{\hbar^{2}}{2 m} \frac{\partial \sqrt{|\psi|^{2}}}{\partial \mathbf{r}} \frac{\partial}{\partial \mathbf{r}} \cdot\left[\frac{1}{\sqrt{|\psi|^{2}}}\right]-\frac{\hbar^{2}}{2 m} \Delta \psi+\frac{\hbar^{2}}{m} \frac{\partial \ln \sqrt{|\psi|^{2}}}{\partial \mathbf{r}} \cdot \frac{\partial \psi}{\partial \mathbf{r}} \\
& -\frac{\hbar^{2}}{2 m} \frac{1}{\psi}\left[\frac{\partial \psi}{\partial \mathbf{r}}\right]^{2}+\frac{i}{\psi} \frac{\hbar^{3}}{2 m^{2}} \frac{\partial}{\partial \mathbf{r}} \cdot\left\{\tau_{q u} \frac{\partial}{\partial \mathbf{r}} \cdot\left[\psi^{2} \frac{\partial \beta}{\partial \mathbf{r}} \frac{\partial \beta}{\partial \mathbf{r}}\right]\right\}-\frac{i}{\psi} \frac{\hbar}{2} \frac{\partial}{\partial \mathbf{r}} \cdot\left\{\tau_{q u} \psi^{2} \mathbf{F}\right\}
\end{aligned}
$$

or

$$
\begin{aligned}
& i \hbar \frac{\partial \psi}{\partial t}-\frac{1}{\psi} i \hbar \frac{\partial}{\partial t}\left\{\tau_{q u} \psi \frac{\partial \psi}{\partial t}\right\}-i \frac{1}{\psi} \frac{\hbar^{2}}{2 m} \frac{\partial}{\partial t}\left\{\tau_{q u} \frac{\partial}{\partial \mathbf{r}} \cdot\left(\psi^{2} \frac{\partial \beta}{\partial \mathbf{r}}\right)\right\} \\
& -\frac{i}{\psi} \frac{\hbar^{2}}{2 m} \frac{\partial}{\partial \mathbf{r}} \cdot\left\{\tau_{q u} \frac{\partial}{\partial t}\left[\psi^{2} \frac{\partial \beta}{\partial \mathbf{r}}\right]\right\} \\
& =-\psi U_{q u}-\psi \frac{\hbar^{2}}{m} \frac{1}{\rho} \frac{\partial \sqrt{|\psi|^{2}}}{\partial \mathbf{r}} \cdot \frac{\partial \sqrt{|\psi|^{2}}}{\partial \mathbf{r}}-\frac{\hbar^{2}}{2 m} \Delta \psi+\frac{\hbar^{2}}{m} \frac{\partial \ln \sqrt{|\psi|^{2}}}{\partial \mathbf{r}} \cdot \frac{\partial \psi}{\partial \mathbf{r}} \\
& -\frac{\hbar^{2}}{2 m} \frac{1}{\psi}\left[\frac{\partial \psi}{\partial \mathbf{r}}\right]^{2}+\frac{i}{\psi} \frac{\hbar^{3}}{2 m^{2}} \frac{\partial}{\partial \mathbf{r}} \cdot\left\{\tau_{q u} \frac{\partial}{\partial \mathbf{r}} \cdot\left[\psi^{2} \frac{\partial \beta}{\partial \mathbf{r}} \frac{\partial \beta}{\partial \mathbf{r}}\right]\right\}-\frac{i}{\psi} \frac{\hbar}{2} \frac{\partial}{\partial \mathbf{r}} \cdot\left\{\tau_{q u} \psi^{2} \mathbf{F}\right\}
\end{aligned}
$$

or

$$
i \hbar \frac{\partial \psi}{\partial t}-\frac{1}{\psi} i \hbar \frac{\partial}{\partial t}\left\{\tau_{q u} \psi \frac{\partial \psi}{\partial t}\right\}-i \frac{1}{\psi} \frac{\hbar^{2}}{2 m} \frac{\partial}{\partial t}\left\{\tau_{q u} \frac{\partial}{\partial \mathbf{r}} \cdot\left(\psi^{2} \frac{\partial \beta}{\partial \mathbf{r}}\right)\right\}
$$




$$
\begin{aligned}
& -\frac{i}{\psi} \frac{\hbar^{2}}{2 m} \frac{\partial}{\partial \mathbf{r}} \cdot\left\{\tau_{q u} \frac{\partial}{\partial t}\left[\psi^{2} \frac{\partial \beta}{\partial \mathbf{r}}\right]\right\} \\
& =-\psi U_{q u}-\psi \frac{\hbar^{2}}{m}\left[\frac{\partial \ln \sqrt{\rho}}{\partial \mathbf{r}}\right]^{2}-\frac{\hbar^{2}}{2 m} \Delta \psi+\frac{\hbar^{2}}{m} \frac{\partial \ln \sqrt{\rho}}{\partial \mathbf{r}} \cdot \frac{\partial \psi}{\partial \mathbf{r}}-\frac{\hbar^{2}}{2 m} \frac{1}{\psi}\left[\frac{\partial \psi}{\partial \mathbf{r}}\right]^{2} \\
& +\frac{i}{\psi} \frac{\hbar^{3}}{2 m^{2}} \frac{\partial}{\partial \mathbf{r}} \cdot\left\{\tau_{q u} \frac{\partial}{\partial \mathbf{r}} \cdot\left[\psi^{2} \frac{\partial \beta}{\partial \mathbf{r}} \frac{\partial \beta}{\partial \mathbf{r}}\right]\right\}-\frac{i}{\psi} \frac{\hbar}{2} \frac{\partial}{\partial \mathbf{r}} \cdot\left\{\tau_{q u} \psi^{2} \mathbf{F}\right\}
\end{aligned}
$$

Let us make some intermediate conclusions:

1) The nonlocal continuity Equation (3.18), written in terms of the wave function and the relations (2.8)-(2.10), is a nonlinear partial differential equation containing derivatives in time, coordinates and cross derivatives.

2) The local continuity equation corresponds to the condition $\tau_{q u}=0$ and has the form

$$
\begin{aligned}
i \hbar \frac{\partial \psi}{\partial t} & =-\frac{\hbar^{2}}{2 m} \Delta \psi-\psi U_{q u} \\
& -\psi \frac{\hbar^{2}}{m}\left[\frac{\partial \ln \sqrt{\rho}}{\partial \mathbf{r}}\right]^{2}+\frac{\hbar^{2}}{m} \frac{\partial \ln \sqrt{\rho}}{\partial \mathbf{r}} \cdot \frac{\partial \psi}{\partial \mathbf{r}}-\frac{\hbar^{2}}{2 m} \frac{\partial \psi}{\partial \mathbf{r}} \cdot \frac{\partial \ln \psi}{\partial \mathbf{r}}
\end{aligned}
$$

3) Equation (3.19) is transformed into the Schrödinger equation

$$
i \hbar \frac{\partial \psi}{\partial t}=-\frac{\hbar^{2}}{2 m} \Delta \psi-\psi U_{q u}
$$

only after discarding the members of the second line in (3.19) as small terms containing derivatives of logarithmic functions. In this case, the nonlinear Equation (3.19) becomes the linear Schrödinger equation.

4) The nonlocal nonlinear Equation (3.18) contains the symmetric cross timecoordinate derivatives (the last two terms of the left hand side of Equation (3.18)).

In the following for the simplicity we use the condition $\tau_{q u}=$ const . We find from Equation (3.18)

$$
\begin{aligned}
& i \hbar \frac{\partial \psi}{\partial t}-\frac{1}{\psi} i \hbar \tau_{q u} \frac{\partial}{\partial t}\left\{\psi \frac{\partial \psi}{\partial t}\right\}-i \tau_{q u} \frac{2}{\psi} \frac{\hbar^{2}}{2 m} \frac{\partial}{\partial t}\left\{\frac{\partial}{\partial \mathbf{r}} \cdot\left(\psi^{2} \frac{\partial \beta}{\partial \mathbf{r}}\right)\right\} \\
& =-\psi U_{q u}-\psi \frac{\hbar^{2}}{m}\left[\frac{\partial \ln \sqrt{\rho}}{\partial \mathbf{r}}\right]^{2}-\frac{\hbar^{2}}{2 m} \Delta \psi+\frac{\hbar^{2}}{m} \frac{\partial \ln \sqrt{\rho}}{\partial \mathbf{r}} \cdot \frac{\partial \psi}{\partial \mathbf{r}}-\frac{\hbar^{2}}{2 m} \frac{1}{\psi}\left[\frac{\partial \psi}{\partial \mathbf{r}}\right]^{2} \\
& +\frac{i}{\psi} \frac{\hbar^{3}}{2 m^{2}} \tau_{q u} \frac{\partial}{\partial \mathbf{r}} \cdot\left\{\frac{\partial}{\partial \mathbf{r}} \cdot\left[\psi^{2} \frac{\partial \beta}{\partial \mathbf{r}} \frac{\partial \beta}{\partial \mathbf{r}}\right]\right\}-\frac{i}{\psi} \tau_{q u} \frac{\hbar}{2} \frac{\partial}{\partial \mathbf{r}} \cdot\left\{\psi^{2} \mathbf{F}\right\}
\end{aligned}
$$

or

$$
\begin{aligned}
& i \hbar \frac{\partial \psi}{\partial t}-i \hbar \tau_{q u} \frac{\partial^{2} \psi}{\partial t^{2}}-\frac{1}{\psi} i \hbar \tau_{q u}\left(\frac{\partial \psi}{\partial t}\right)^{2}-i \tau_{q u} \frac{2}{\psi} \frac{\hbar^{2}}{2 m} \frac{\partial}{\partial t}\left\{\frac{\partial}{\partial \mathbf{r}} \cdot\left(\psi^{2} \frac{\partial \beta}{\partial \mathbf{r}}\right)\right\} \\
& =-\psi U_{q u}-\psi \frac{\hbar^{2}}{m}\left[\frac{\partial \ln \sqrt{\rho}}{\partial \mathbf{r}}\right]^{2}-\frac{\hbar^{2}}{2 m} \Delta \psi+\frac{\hbar^{2}}{m} \frac{\partial \ln \sqrt{\rho}}{\partial \mathbf{r}} \cdot \frac{\partial \psi}{\partial \mathbf{r}}-\frac{\hbar^{2}}{2 m} \frac{1}{\psi}\left[\frac{\partial \psi}{\partial \mathbf{r}}\right]^{2} \\
& +\frac{i}{\psi} \frac{\hbar^{3}}{2 m^{2}} \tau_{q u} \frac{\partial}{\partial \mathbf{r}} \cdot\left\{\frac{\partial}{\partial \mathbf{r}} \cdot\left[\psi^{2} \frac{\partial \beta}{\partial \mathbf{r}} \frac{\partial \beta}{\partial \mathbf{r}}\right]\right\}-\frac{i}{\psi} \tau_{q u} \frac{\hbar}{2} \frac{\partial}{\partial \mathbf{r}} \cdot\left\{\psi^{2} \mathbf{F}\right\}
\end{aligned}
$$


Let us consider the penultimate term of Equation (3.22) using the tensor relations

$$
\mathbf{a b}: \mathbf{c d}=(\mathbf{a} \cdot \mathbf{d})(\mathbf{b} \cdot \mathbf{c})
$$

and

$$
\mathbf{b} \cdot(\mathbf{a} \cdot \mathrm{W})=\mathbf{b a}: \mathrm{W},
$$

where the double point $(:)$ denotes the double tensor product. In this case

$$
\begin{aligned}
\frac{\partial}{\partial \mathbf{r}} \cdot\left\{\frac{\partial}{\partial \mathbf{r}} \cdot\left[\psi^{2} \frac{\partial \beta}{\partial \mathbf{r}} \frac{\partial \beta}{\partial \mathbf{r}}\right]\right\} & =\frac{\partial}{\partial \mathbf{r}} \frac{\partial}{\partial \mathbf{r}}: \psi^{2} \frac{\partial \beta}{\partial \mathbf{r}} \frac{\partial \beta}{\partial \mathbf{r}}=\left(\frac{\partial}{\partial \mathbf{r}} \cdot \frac{\partial \beta}{\partial \mathbf{r}}\right)\left(\frac{\partial}{\partial \mathbf{r}} \cdot \psi^{2} \frac{\partial \beta}{\partial \mathbf{r}}\right) \\
& =\left(\frac{\partial}{\partial \mathbf{r}} \cdot \frac{\partial \beta}{\partial \mathbf{r}}\right)\left[\psi^{2} \frac{\partial}{\partial \mathbf{r}} \cdot \frac{\partial \beta}{\partial \mathbf{r}}+2 \psi \frac{\partial \beta}{\partial \mathbf{r}} \cdot \frac{\partial \psi}{\partial \mathbf{r}}\right]
\end{aligned}
$$

Then Equation (3.22) takes the form

$$
\begin{aligned}
& i \hbar \frac{\partial \psi}{\partial t}-i \hbar \tau_{q u} \frac{\partial^{2} \psi}{\partial t^{2}}-\frac{1}{\psi} i \hbar \tau_{q u}\left(\frac{\partial \psi}{\partial t}\right)^{2}-i \tau_{q u} \frac{2}{\psi} \frac{\hbar^{2}}{2 m} \frac{\partial}{\partial t}\left\{\frac{\partial}{\partial \mathbf{r}} \cdot\left(\psi^{2} \frac{\partial \beta}{\partial \mathbf{r}}\right)\right\} \\
& =-\psi U_{q u}-\psi \frac{\hbar^{2}}{m}\left[\frac{\partial \ln \sqrt{\rho}}{\partial \mathbf{r}}\right]^{2}-\frac{\hbar^{2}}{2 m} \Delta \psi+\frac{\hbar^{2}}{m} \frac{\partial \ln \sqrt{\rho}}{\partial \mathbf{r}} \cdot \frac{\partial \psi}{\partial \mathbf{r}}-\frac{\hbar^{2}}{2 m} \frac{1}{\psi}\left[\frac{\partial \psi}{\partial \mathbf{r}}\right]^{2} \\
& +i \frac{\hbar^{3}}{2 m^{2}} \tau_{q u}\left\{\left(\frac{\partial}{\partial \mathbf{r}} \cdot \frac{\partial \beta}{\partial \mathbf{r}}\right)\left[\psi \frac{\partial}{\partial \mathbf{r}} \cdot \frac{\partial \beta}{\partial \mathbf{r}}+2 \frac{\partial \beta}{\partial \mathbf{r}} \cdot \frac{\partial \psi}{\partial \mathbf{r}}\right]\right\}-\frac{i}{\psi} \tau_{q u} \frac{\hbar}{2} \frac{\partial}{\partial \mathbf{r}} \cdot\left\{\psi^{2} \mathbf{F}\right\}
\end{aligned}
$$

or using the relation (3.6) we reach

$$
\begin{aligned}
& i \hbar \frac{\partial \psi}{\partial t}-i \hbar \tau_{q u} \frac{\partial^{2} \psi}{\partial t^{2}}-i \hbar \tau_{q u} \frac{\partial \psi}{\partial t} \frac{\partial \ln \psi}{\partial t} \\
& +\tau_{q u} \frac{1}{\psi} \frac{\hbar^{2}}{m} \frac{\partial}{\partial t}\left\{\frac{\partial}{\partial \mathbf{r}} \cdot\left(\psi^{2}\left(\frac{\partial \ln \sqrt{|\psi|^{2}}}{\partial \mathbf{r}}-\frac{\partial \ln \psi}{\partial \mathbf{r}}\right)\right)\right\} \\
& =-\psi U_{q u}-\frac{\hbar^{2}}{2 m} \Delta \psi-\psi \frac{\hbar^{2}}{m}\left[\frac{\partial \ln \sqrt{\rho}}{\partial \mathbf{r}}\right]^{2}+\frac{\hbar^{2}}{m} \frac{\partial \ln \sqrt{\rho}}{\partial \mathbf{r}} \cdot \frac{\partial \psi}{\partial \mathbf{r}} \\
& -\frac{\hbar^{2}}{2 m} \frac{\partial \ln \psi}{\partial \mathbf{r}} \cdot \frac{\partial \psi}{\partial \mathbf{r}}-i \frac{\hbar^{3}}{2 m^{2}} \tau_{q u} \psi\left[\frac{\partial}{\partial \mathbf{r}} \cdot\left(\frac{\partial \ln \sqrt{|\psi|^{2}}}{\partial \mathbf{r}}-\frac{\partial \ln \psi}{\partial \mathbf{r}}\right)\right]^{2} \\
& -i \frac{\hbar^{3}}{m^{2}} \tau_{q u}\left(\frac{\partial}{\partial \mathbf{r}} \cdot\left(\frac{\partial \ln \sqrt{|\psi|^{2}}}{\partial \mathbf{r}}-\frac{\partial \ln \psi}{\partial \mathbf{r}}\right)\right)\left(\frac{\partial \ln \sqrt{|\psi|^{2}}}{\partial \mathbf{r}}-\frac{\partial \ln \psi}{\partial \mathbf{r}}\right) \cdot \frac{\partial \psi}{\partial \mathbf{r}} \\
& -\frac{i}{\psi} \tau_{q u} \frac{\hbar}{2} \frac{\partial}{\partial \mathbf{r}} \cdot\left(\psi{ }^{2} \mathbf{F}\right)
\end{aligned}
$$

Omitting all terms containing the derivatives of the logarithmic functions we have

$$
i \hbar \frac{\partial \psi}{\partial t}-i \hbar \tau_{q u} \frac{\partial^{2} \psi}{\partial t^{2}}=-\psi U_{q u}-\frac{\hbar^{2}}{2 m} \Delta \psi-\frac{i}{\psi} \tau_{q u} \frac{\hbar}{2} \frac{\partial}{\partial \mathbf{r}} \cdot\left(\psi^{2} \mathbf{F}\right)
$$

or

$$
i \hbar \frac{\partial \psi}{\partial t}-i \hbar \tau_{q u} \frac{\partial^{2} \psi}{\partial t^{2}}=-\left[\frac{\hbar^{2}}{2 m} \Delta+U_{q u}\right] \psi-i \hbar \tau_{q u} \psi \frac{1}{2} \frac{\partial}{\partial \mathbf{r}} \cdot \mathbf{F}-i \hbar \tau_{q u} \mathbf{F} \cdot \frac{\partial \psi}{\partial \mathbf{r}} .
$$


Omitting all terms containing nonlocal parameter $\tau_{q u}$ in Equation (3.29) we lose the last connection with non-local physics and find the typical form of the Schrödinger equation

$$
i \hbar \frac{\partial \psi}{\partial t}=-\left[\frac{\hbar^{2}}{2 m} \Delta+U_{q u}\right] \psi=0,
$$

where

$$
U_{q u}=-\frac{\hbar^{2}}{2 m \sqrt{\rho}} \Delta \sqrt{\rho} .
$$

is the quantum potential in the Bohm interpretation.

I underline that this equation does not contain the nonlocal parameter $\tau_{q u}$ at all. It means that from the position of nonlocal physics the linear Schrödinger equation is local equation. This fact creates all difficulties of the Schrödinger wave mechanics.

Interesting to notice, that introducing the operator of the substation derivative

$$
\frac{D}{D t}=\frac{\partial}{\partial t}+\tau_{q u} \mathbf{F} \cdot \frac{\partial}{\partial \mathbf{r}},
$$

we obtain the kinetic transport equation with the relativistic correction in the form

$$
\frac{D \psi}{D t}-\tau_{q u} \frac{\partial^{2} \psi}{\partial t^{2}}=-\frac{1}{i \hbar}\left[\frac{\hbar^{2}}{2 m} \Delta+U_{q u}\right] \psi-\tau_{q u} \psi \frac{1}{2} \frac{\partial}{\partial \mathbf{r}} \cdot \mathbf{F}
$$

or

$$
\frac{D \psi}{D t}-\tau_{q u} \frac{\partial^{2} \psi}{\partial t^{2}}=i\left[\frac{\hbar}{2 m} \Delta+\frac{1}{\hbar} U_{q u}\right] \psi-\tau_{q u} \psi \frac{1}{2} \frac{\partial}{\partial \mathbf{r}} \cdot \mathbf{F}
$$

or

$$
\frac{D \psi}{D t}-\tau_{q u} \frac{\partial^{2} \psi}{\partial t^{2}}=\left[\frac{i \hbar}{2 m} \Delta+\frac{i}{\hbar} U_{q u}-\tau_{q u} \frac{1}{2} \frac{\partial}{\partial \mathbf{r}} \cdot \mathbf{F}\right] \psi
$$

The force $\mathbf{F}$ (in Equation (3.28)) is the force acting on the unit mass. In the theory of superconductivity, we can use $\mathbf{F}=\frac{e}{m} \mathbf{E}$. Then

$$
\frac{\partial}{\partial \mathbf{r}} \cdot \mathbf{F}=\frac{e}{m} \frac{\partial}{\partial \mathbf{r}} \cdot \mathbf{E}=\frac{e}{m} \rho_{e},
$$

where $\rho_{e}$ is the charge density. Then using (3.35) and (3.36) we obtain

$$
\frac{D \psi}{D t}-\tau_{q u} \frac{\partial^{2} \psi}{\partial t^{2}}=\left[\frac{i \hbar}{2 m} \Delta+\frac{i}{\hbar} U_{q u}-\tau_{q u} \frac{e}{2 m} \rho_{e}\right] \psi
$$

or

$$
\frac{D \psi}{D t}-\tau_{q u} \frac{\partial^{2} \psi}{\partial t^{2}}=\left[\frac{i \hbar}{2 m} \Delta+\frac{i}{\hbar} U_{q u}-\tau_{q u} \frac{e}{2 m}|\psi|^{2}\right] \psi
$$

\section{Conclusions}

1) For the SE obtaining we need only a generalized continuity equation even 
without generalized motion equation and generalized energy equation. Moreover, the Schrödinger equation corresponds to the generalized continuity equation with nonlocal parameter $\tau_{q u}=0$. But we need to omit all nonlinear terms containing the derivatives of logarithmic functions.

2) Omitting nonlinear terms in the generalized continuity equation but conserving the terms proportional to $\tau_{q u}$ we obtain other known quantum equations. In particular, the second term in the left-hand side of Equation (3.29) corresponds to the relativistic Schrödinger equation. The corresponding last terms in Equation (3.38) lead to the Ginzburg-Landau theory.

3) A rhetorical question arises-why does the Schrödinger equation work at all? Violation of the Bell inequalities for local statistical theories is established and the transition to a nonlocal description is inevitable.

The physics of the twenty-first century is nonlocal physics.

\section{Acknowledgements}

My thanks are to Dr. Alex Fedoseyev for the fruitful discussions.

\section{Conflicts of Interest}

The author declares no conflicts of interest regarding the publication of this paper.

\section{References}

[1] Boltzmann, L. (1872) Weitere Studien über das Wärmegleichgewicht unter Gasmolekulen. Sitz. Ber. Kaiserl. Akad. Wiss, 66, s.275.

[2] Alekseev, B.V. (1982) Matematicheskaya Kinetika Reagiruyushchikh Gazov (Mathematical Theory of Reacting Gases). Nauka, Moscow.

[3] Alexeev, B.V. (2004) Generalized Boltzmann Physical Kinetics. Elsevier, Amsterdam, 368 p. https://doi.org/10.1016/B978-044451582-7/50027-8

[4] Alexeev, B.V. (2015) Unified Non-Local Theory of Transport Processes. Elsevier, Amsterdam, $644 \mathrm{p}$.

[5] Alexeev, B.V. (2016) Unified Non-Local Relativistic Theory of Transport Processes. Elsevier, Amsterdam, 455 p.

[6] Alexeev, B.V. (2017) Nonlocal Astrophysics. Dark Matter, Dark Energy, Physical Vacuum. Elsevier, Amsterdam, 454 p.

[7] Alexeev, B.V. (1994) The Generalized Boltzmann Equation, Generalized Hydrodynamic Equations and Their Applications. Philosophical Transactions of the Royal Society A, 349, 417-443. https://doi.org/10.1098/rsta.1994.0140

[8] Alexeev, B.V. (1995) The Generalized Boltzmann Equation. Physica A, 216, 459-468. https://doi.org/10.1016/0378-4371(95)00044-8

[9] Alexeev, B.V. (2008) Generalized Quantum Hydrodynamics and Principles of Non-Local Physics. Journal of Nanoelectronics and Optoelectronics, 3, 143-158. https://doi.org/10.1166/jno.2008.207

[10] Alexeev, B.V. (2008) Application of Generalized Quantum Hydrodynamics in the Theory of Quantum Soliton's Evolution. Journal of Nanoelectronics and Optoelectronics, 3, 316-328. https://doi.org/10.1166/jno.2008.311 
[11] Madelung, E. (1927) Quantentheorie in Hydrodynamischer Form. Zeitschrift für physic, 40, 322-326. https://doi.org/10.1007/BF01400372

[12] Bell, J.S. (1964) On the Einstein Podolsky Rosen Paradox. Physics, 1, Article No. 195. https://doi.org/10.1103/PhysicsPhysiqueFizika.1.195

[13] Wigner, E. (1961) Group Theory and Its Applications to the Quantum Mechanical Theory of Atomic Spectra.

[14] Schrödinger, E. (1926) Quantization as an Eigenvalue Problem. Annalen der Physik, 79, Article No. 361.

[15] Abramowitz, M. and Stegun, I.A. (1964) Handbook of Mathematical Functions. Dover Publications, New York, 74.

[16] Alexeev, B.V. (2018) Extremal States in Nonlocal Physics. Lambert Academic Publishing, Saarbrücken.

[17] Alexeev, B.V. (2021) Nonlocal Theory of High-Temperature Superconductivity. Journal of Modern Physics, 12, 552-593. https://www.scirp.org/journal/jmp https://doi.org/10.4236/jmp.2021.125037

[18] Bohm, D. (1952) Quantum Theory. Prentice-Hall, Inc., New York.

[19] Alexeev, B.V. (2013) Application of the Non-Local Physics in the Theory of Gravitational Waves and Big Bang. Journal of Modern Physics, 4, 26-41.

[20] Alexeev, B.V. (2013) Application of the Non-Local Physics in the Theory of the Matter Movement in Black Hole. Journal of Modern Physics, 4, 42-49.

https://doi.org/10.4236/jmp.2013.47A1005 